\title{
Higgs boson decays into narrow diphoton jets and their search strategies at the Large Hadron Collider
}

\author{
Benjamin Sheff®, Noah Steinberg $\odot$, and James D. Wells๑ \\ Leinweber Center for Theoretical Physics Physics Department, University of Michigan, \\ Ann Arbor, Michigan 48109-1040, USA
}

(Received 3 December 2020; accepted 22 July 2021; published 24 August 2021)

\begin{abstract}
In many extensions of the Standard Model, the Higgs boson can decay into two light scalars, each of which then subsequently decay into two photons. The underlying event is $h \rightarrow 4 \gamma$, but the kinematics from boosted light scalar decays combined with realistic detector resolutions may fail to register the events in straightforward categories and thus may be lost. In this article, we investigate the phase space for highly boosted diphoton events from these exotic Higgs decays and discuss search strategies that aim to capture and label events in this difficult region. In the process, we develop a new category, $\xi$ jets, which identifies with high selectivity highly collimated diphoton decay modes of the Higgs boson.
\end{abstract}

DOI: 10.1103/PhysRevD.104.036009

\section{INTRODUCTION}

Nearly a decade after the discovery of the Higgs boson, it remains to be decided whether the discovered particle interacts with other known elementary particles in precisely the way the Standard Model (SM) dictates [1-6]. Deviations from SM expectations can arise by virtue of the Higgs boson being composite, part of a larger Higgs sector, coupled through its portal interactions to hidden sector states, or embedded in extra dimensions to name just a few examples. Alternatives remain viable because the SM Higgs boson couplings to other SM states are known only to at best $10 \%$ for some and only to within $\mathcal{O}(1)$ factors for others, including muon, electron, charm, and Higgs self-interactions $[7,8]$. The possibility of the Higgs boson decaying into final states that are not allowed by the SM is also not constrained well in many cases.

In this article, we take up the case of the Higgs boson $(h)$ decaying into other very light scalars $\left(\phi_{1}\right.$ and $\left.\phi_{2}\right)$ where each subsequently decays into photons,

$$
h \rightarrow \phi_{1} \phi_{2} \rightarrow(\gamma \gamma)(\gamma \gamma) \quad \text { (target observable). }
$$

In several different limits, this process has been studied already $[9,10]$. In the case of $\phi_{1,2}$ both having mass above about $10 \mathrm{GeV}$, one finds that the events register as unambiguous $4 \gamma$ events in the detector that can be searched

Published by the American Physical Society under the terms of the Creative Commons Attribution 4.0 International license. Further distribution of this work must maintain attribution to the author(s) and the published article's title, journal citation, and DOI. Funded by SCOAP ${ }^{3}$. for well. Within this regime, current studies limit this process to $B(h \rightarrow 4 \gamma) \lesssim 3 \times 10^{-4}[11,12]$.

On the other extreme, if $\phi_{1,2}$ both have mass less than a few hundred $\mathrm{MeV}$, the photons from $\phi_{i} \rightarrow \gamma \gamma$ are so collimated coming from the highly boosted $\phi_{i}$ resultant from their parent Higgs decay that each $\phi_{i}$ decay appears to go to a single photon. In that case, $h \rightarrow \phi_{1} \phi_{2}$ is simply combined with the standard $h \rightarrow \gamma \gamma$ analysis, and it becomes a statistical question to determine what overabundance of such a signal would be consistent with data. At 95\% C.L., the answer to this question is that the branching fraction of non-SM contributions to $B(h \rightarrow \gamma \gamma)$ cannot exceed $2.2 \times 10^{-4}$ [13]. Such light scalars may also be disentangled from the SM $h \rightarrow \gamma \gamma$ process with sophisticated substructure techniques $[14,15]$.

Combining both extremes leads to an apparent detection $h \rightarrow 3 \gamma$. This arises when one of the $\phi_{i}$ has mass less than a few hundred $\mathrm{MeV}$ and the other has more than about $10 \mathrm{GeV}$. This process is forbidden in the $\mathrm{SM}$, and the branching ratio is currently limited to $B(h \rightarrow 3 \gamma) \lesssim \times 10^{-3}$, as can be gleaned from Ref. [16].

In between these two extremes, from the point of view of observables, is a murky region where the mass of one or both $\phi_{i}$ states is between approximately 0.1 and $10 \mathrm{GeV}$. In that case, the two photons coming out of the $\phi_{i}$ decays are not highly collimated; nor are they cleanly separated. Roughly speaking, the ATLAS and CMS detectors see something distinct from a standard photon but that also does not register as two photons when the photon separation is between $0.04<\Delta R<0.4[17,18]$. It is this difficult middle ground region that we wish to address in this paper.

It should be stated that extending the scalar sector of the SM by one (or multiple) singlets is a mature and wellstudied subfield [19-21]. Much of the parameter space for 
exotic heavy and light scalars (relative to the Higgs boson mass) is well constrained by direct searches and by precision electroweak measurements [22]. Our simplified model highlights a region of parameter space in a class of singlet extended models that has been less explored by previous studies.

The value in exploring such a regime lies in its ability to utilize the available experimental power from the LHC to investigate one of the most interesting loose ends in the Standard Model. Many models exist that couple new light scalars to the Standard Model in ways that are highly susceptible to the search strategy we advocate here [23]. The nature of the Higgs boson makes such couplings to new physics generic and apparent in a broad swath of theory parameter space. Furthermore, the rough knowledge we have of the Higgs boson to date deserves significant tightening in every reasonable direction. Our goal here is to consider this particular case in detail, highlight the experimental challenges for discovery, proffer some suggestions, and suggest a benchmark theory with points that may be useful for serious further study by experimental groups within the ATLAS and CMS collaborations.

\section{THEORY DESCRIPTION}

The phenomenon we are after is $h \rightarrow \phi_{1} \phi_{2}$ with subsequent decay of $\phi_{i} \rightarrow \gamma \gamma$. Such decays arise generically in a broad class of BSM theories, many of which give rise to additional exotic phenomena. Most commonly, these are other, similar gauge interactions, such as $Z \rightarrow \phi \gamma$, but the possibilities are wide and varied. Many BSM theories of this type are not yet constrained by experiment and have their most accessible phenomenon as $h \rightarrow \phi_{1} \phi_{2} \rightarrow 4 \gamma$, if there are dedicated searches for it. Our focus lies in this last type of theory.

To devise an experimental strategy and analysis to discover this class of targeted theories, we must begin by constructing a representative theory within the class and finding ways to find evidence for it. Ideally, the representative theory should be maximally simple without losing the key features under consideration for our exotic Higgs decays. In this case, there is such a simple theory, and its Lagrangian is

$$
\begin{aligned}
\mathcal{L}= & \mathcal{L}_{\mathrm{SM}}+\frac{1}{2}\left(\partial_{\mu} \phi_{1}\right)\left(\partial^{\mu} \phi_{1}\right)+\frac{1}{2}\left(\partial_{\mu} \phi_{2}\right)\left(\partial^{\mu} \phi_{2}\right)-\frac{1}{2} m_{1}^{2} \phi_{1}^{2} \\
& -\frac{1}{2} m_{2}^{2} \phi_{2}^{2}+\lambda_{\phi}|H|^{2} \phi_{1} \phi_{2}+\frac{1}{\Lambda_{1}} \phi_{1} F_{\mu \nu} F^{\mu \nu} \\
& +\frac{1}{\Lambda_{2}} \phi_{2} F_{\mu \nu} F^{\mu \nu} \quad \text { (representative theory) }
\end{aligned}
$$

where $F^{\mu \nu}$ is the photon field strength tensor. Of course, one could write down nontrivial $|H|^{2} \phi_{1}^{2}$ and $|H|^{2} \phi_{2}^{2}$ terms among others, but that would add complexity without contributing significantly to the final phenomenology.
One might also object that $\phi_{i} F_{\mu \nu} F^{\mu \nu}$ should be traded in for gauge-invariant couplings of $\phi_{i}$ to hypercharge field strength tensor $\phi_{i} B_{\mu \nu} B^{\mu \nu}$ and $S U(2)$ field strength tensor $\phi_{i} W_{\mu \nu}^{a} W^{a, \mu \nu}$. That would be fine, except that upon diagonalizing these interactions to those of the mass eigenstates one finds nevertheless $\phi_{i} F^{2}$ terms, which will completely dominate in the decays of $\phi_{i}$ over $\phi_{i} Z^{\mu \nu} F_{\mu \nu}$ and $\phi_{i} Z^{2}$ terms due to the $Z$ boson being much heavier than the $\phi_{i}$ that we will consider below. ${ }^{1}$ The $\phi_{i} Z F$ interaction can give rise to $Z \rightarrow \phi_{i} \gamma$ decays, constrained by searches at the Tevatron and the LHC $[11,24]$, but as the scale of $\Lambda_{i}$ becomes higher, this constraint goes away, while $B\left(\phi_{i} \rightarrow \gamma \gamma\right)$ remains $100 \% .^{2}$ For that reason, we drop these extra considerations and extraneous interactions from the theory description and retain only the Lagrangian of Eq. (2).

From the point of view of devising experimental search strategies to find evidence for the Higgs boson decaying into a single light scalar, say, $\sigma$ such that $h \rightarrow \sigma \sigma \rightarrow 4 \gamma$, the benchmark theory above is adequate. It merely corresponds to the case of $m_{1}=m_{2}$. That is not to say the two theories are exactly the same, only that the subsequent search strategies are the same. That is why we propose to work with only one theory-the representative theory of Eq. (2) — which we believe to form a basis upon which benchmark points can be established and strategies devised.

\section{PHOTON $\xi$ JETS}

As we mentioned in the Introduction, the target observable of Eq. (1) implies photon separation from $\phi_{i}$ decays that is sensitive to the $\phi_{i}$ masses. This separation is given by

$$
\Delta R_{\gamma \gamma} \sim 2 m_{\phi_{i}} / E_{\phi_{i}} \sim 4 m_{\phi_{i}} / m_{h}
$$

This is illustrated in Fig. 1, which shows that $m_{\phi}=10 \mathrm{GeV}$ gives well-separated photons $(\Delta R>0.4)$ and $m_{\phi}=0.1 \mathrm{GeV}$ gives very collimated photons $(\Delta R<0.04)$, and a mass of $1 \mathrm{GeV}$ gives intermediate separation. Recall that $\Delta R=\sqrt{(\Delta \phi)^{2}+(\Delta \eta)^{2}}$, and $\Delta \phi$ is the azimuthal angle separation, and $\Delta \eta$ is the pseudorapidity separation of the two photons in $\phi_{i} \rightarrow \gamma \gamma$ decay.

We turn to the ambiguous case in which the $\phi_{i}$ masses fall within the "intermediate mass" range of $1 \mathrm{GeV}<$ $m_{\phi_{i}}<10 \mathrm{GeV}$. Within the LHC environment, the production of Higgs bosons and their subsequent decay into such scalars yields photon pairs separated by

\footnotetext{
${ }^{1}$ Barring any tuned cancellations, typical branching ratios to $\gamma \gamma$ are $10^{7}\left(10^{15}\right)$ times larger than the branching ratios to $\gamma Z^{*}\left(Z^{*} Z^{*}\right)$

${ }^{2}$ As the $\Lambda_{i}$ increase, so does the decay length of the scalar. We have checked that the scalar decay length can be under $1 \mathrm{~mm}$ even for large $(\mathrm{PeV})$ values of $\Lambda_{i}$ which evade the $Z \rightarrow \phi_{i} \gamma$ constraint.
} 


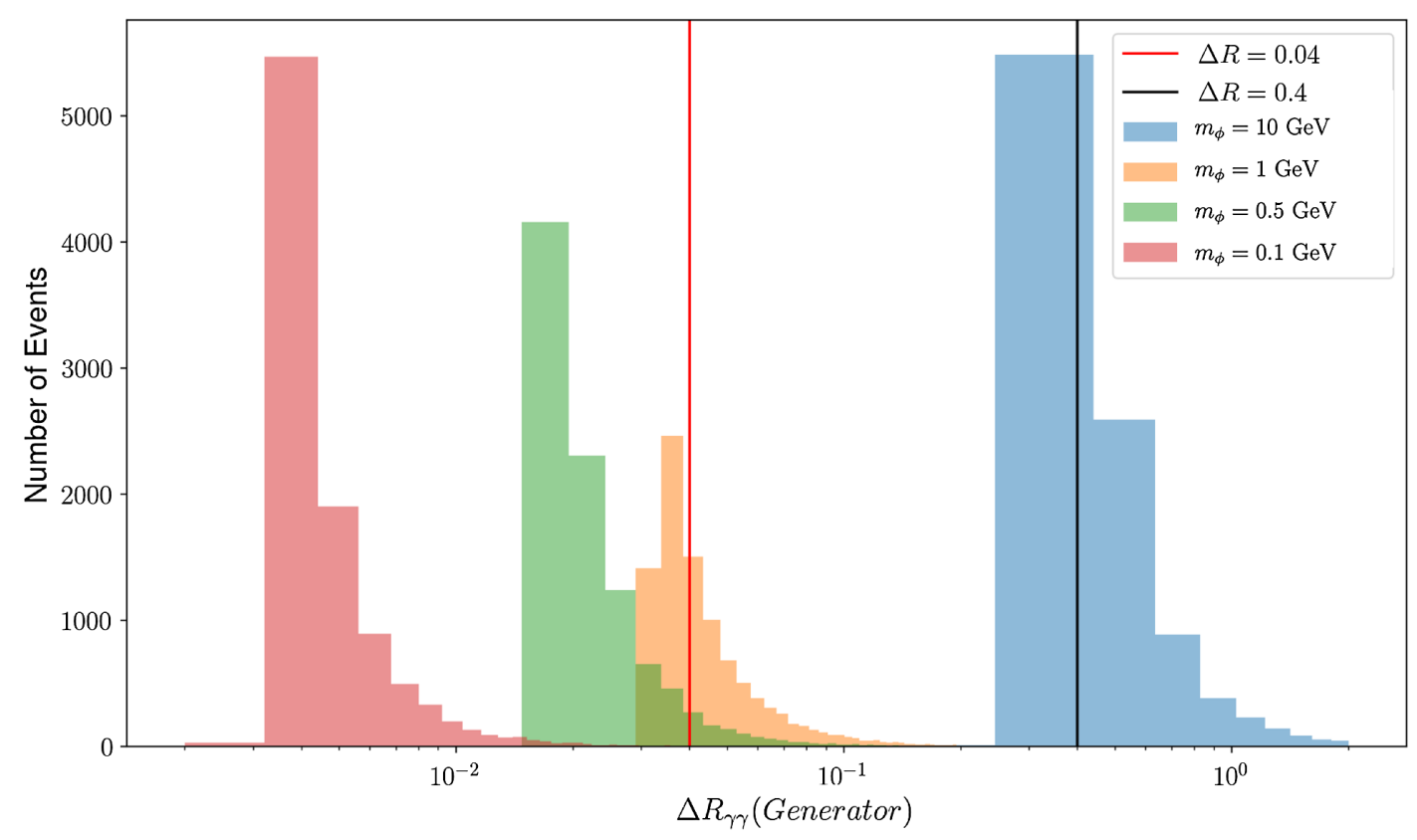

FIG. 1. $\Delta R$ separation between photon pairs from $\phi_{i}$ decays, sampled over 10,000 events at varying masses of the BSM scalars.

$$
0.04<\Delta R_{\gamma \gamma}<0.4 \quad \text { (intermediate separation) }
$$

It is well known that photon pairs that fall within the intermediate separation range of Eq. (4) are extremely difficult to separate or identity. We will speak much more on that below, but here we wish to pay respect to that difficulty by giving it a name. We call two photons that are within the range specified by Eq. (4) a " $\xi$ jet." The $\xi$ jet is a purely theoretical object, and it is defined by underlying "truth data" and not with respect to any detector performance. If a photon has another photon within the intermediate separation annulus of Eq. (4), and nothing else is within the outer ring of that annulus, then it ceases to be a photon, and the two together form a $\xi$ jet. Such a concept can be generalized to more than two photons, but it is of not much importance here to do that. We also specify as a theoretical object that a photon is defined to be either a single photon or two photons within $\Delta R<0.04$ of each other.

With these theory definitions of the photon and $\xi$ jet, our target observable is broken into several distinct and nonoverlapping final states, depending on the masses of the $\phi_{i}$ intermediate states in the decay chain:

$$
\begin{aligned}
h & \rightarrow \phi_{1} \phi_{2} \rightarrow 4 \gamma \\
& \Rightarrow 4 \gamma, 2 \gamma, 3 \gamma, \gamma \xi, \gamma \gamma \xi, 2 \xi \quad \text { (observable partitions). }
\end{aligned}
$$

The first three of these observables we have already discussed. The remaining observables have not been fully explored in the literature, and we wish to consider them in more detail below.

\section{BENCHMARK MODEL POINTS}

We are interested in exploring three observables: $\gamma \xi, \gamma \gamma \xi$, and $2 \xi$. To do so, we need benchmark points that give rise to each of these types of observables. They can be obtained rather straightforwardly from our representative theory of Eq. (2) where the masses of $\phi_{1}$ and $\phi_{2}$ are chosen to be various permutations of the masses $0.1,1$, and $10 \mathrm{GeV}$. In particular, $m_{\phi}=0.1 \mathrm{GeV}$ generally always gives $\phi \rightarrow \gamma$ decays, $m_{\phi}=1 \mathrm{GeV}$ typically gives $\phi \rightarrow \xi$ decays, and $m_{\phi}=10 \mathrm{GeV}$ generally gives $\phi \rightarrow \gamma \gamma$ decays according to our definitions in the previous section. These are so far entirely defined theoretically. In the next section, we will pursue more carefully how a theoretical $\xi$ jet registers in an experimental analysis.

From these considerations, we can construct the four benchmark points A, B, C, and D specified in Table I. Figure 2 shows the relative fraction of each observable for each benchmark point. The dominant and subdominant

TABLE I. Benchmark points for $h \rightarrow \phi_{1} \phi_{2} \rightarrow 4 \gamma$, which then partition into various theory-object observables (modes) according to our definitions of $\xi$ (photon pairs with $0.04<\Delta R<0.4$ ) and $\gamma$ (an isolated photon with $\Delta R>0.4$ or two photons within $\Delta R<0.04)$.

\begin{tabular}{lcccc}
\hline \hline Point & $\begin{array}{c}m_{1} \\
(\mathrm{GeV})\end{array}$ & $\begin{array}{c}m_{2} \\
(\mathrm{GeV})\end{array}$ & $\begin{array}{c}\text { Dominant } \\
\text { mode }\end{array}$ & $\begin{array}{c}\text { Subdominant } \\
\text { mode }\end{array}$ \\
\hline A & 1 & 10 & $\gamma \gamma \xi$ & $\gamma \xi \simeq 2 \xi \simeq 3 \gamma$ \\
B & 0.1 & 1 & $\gamma \xi$ & $2 \gamma$ \\
C & 1 & 1 & $2 \xi$ & $\gamma \xi \simeq 2 \gamma$ \\
D & 0.1 & 10 & $3 \gamma$ & $\gamma \xi$ \\
\hline \hline
\end{tabular}




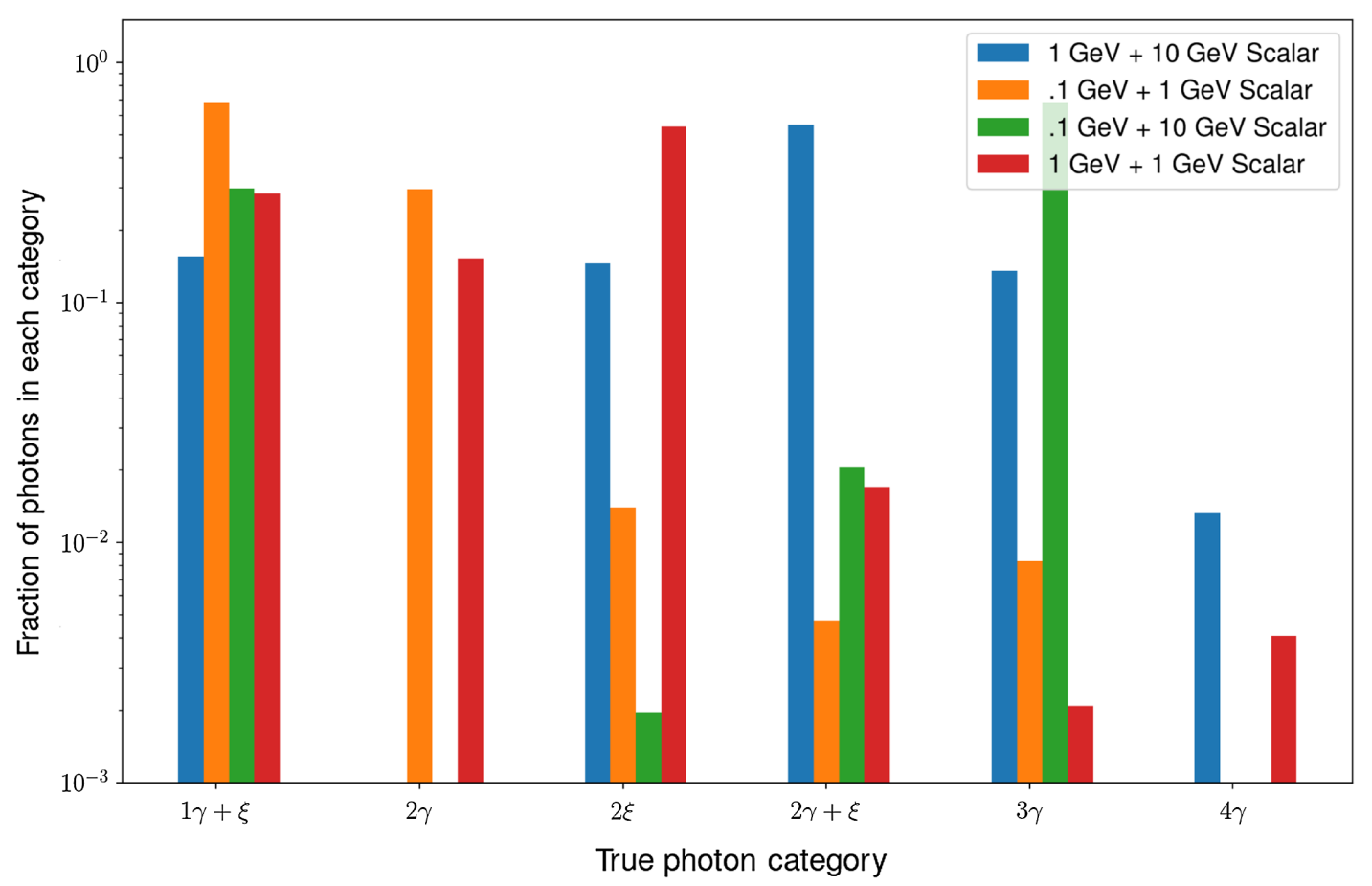

FIG. 2. Branching fraction into each final state theory observable for the benchmark points A (blue), B (orange), C (red), and D (green) given in Table I.

modes of decay for each benchmark point are listed in Table I and can be gleaned from the fraction data given in Fig. 2. Table I shows that several combinations of light scalar masses give interesting decay signatures involving combinations of $\xi$ jets and photons which (to the best of our knowledge) are not being searched for in current LHC analyses.

\section{EXPERIMENTAL SEARCH STRATEGIES}

So far, our discussion has been mainly theoretical. We have identified a rare Higgs decay whose cascade we claim may be difficult to detect by experiment. In this section, we discuss how our theoretical objects translate into experimental manifestations. We have suggested that some mass ranges of $\phi_{i}$ are problematic for experiment. We will discuss some details on why they are challenging and some strategies by which to possibly overcome those challenges.

\section{A. Multiphoton final states}

Isolated photons or extremely highly collimated photons both get identified simply as photons, and analysis based on those standard objects (photons) proceed without much subtlety regarding how to process the data into well-defined final states of $2 \gamma, 3 \gamma$, and $4 \gamma$.

\section{B. $\boldsymbol{\xi}$ jet final states}

Some of the final states from the decays of Eq. (5) yield $\xi$ jets. Underneath, a $\xi$ jet is merely two photons with intermediate $\Delta R$ separation [see Eq. (4)]. But a key question is how a $\xi$ jet, defined as a theoretical object, gets processed into various experimental categories. A perfect detector would register it as merely two photons; a bad detector registers it as a single photon or nothing; and a realistic good detector, such as ATLAS or CMS, registers it as something altogether different within several possible categories of varying sensitivity and selectivity. ${ }^{3}$

To address this question of how a $\xi$ registers in a detector, it is useful to describe the various categories into which a single photon can fall. For an example, we take the standard categories which ATLAS uses for photon identification. There are eight possible standard categories: six are the permutations among three isolation possibilities (nonisolated, loose isolation, and tight isolation) and two ID possibilities (loose ID and tight ID), and the other two categories are jet and lost. Jet is the standard QCD jet from fragmentation of quarks or gluons, and "lost" refers to the possibility that the data do not conform to any other category and are not registered in any higher abstracted category except for mere energy depositions in the detector.

A $\xi$ jet will register with some probability into one or more of the standard photon categories. The probability to

\footnotetext{
${ }^{3}$ By perfect (nonexistent) "sensitivity," we mean a category test that passes with $100 \%(0 \%)$ rate if the underlying event is a $\xi$ jet, and by perfect (nonexistent) "selectivity," we mean a category test that passes with $0 \%(100 \%)$ rate if the underlying event is not a $\xi$ jet. Good sensitivity means a low false negative rate, and good selectivity means a low false positive rate.
} 
do so depends on the underlying event kinematics. Under typical assumptions, the $\xi$ jet will often register as lost due to the inability to resolve the two photons, yet the event covers more than one cell in the electromagnetic calorimeter, which a single photon would not do. As no category becomes applicable, it has no option but to be relegated to lost.

The implication of a $\xi$ jet arising from a Higgs decay being categorized as lost is that an analysis that requires reconstructing the invariant mass of the Higgs boson from well-defined decay products can no longer register the events. It is therefore necessary to build a ninth category " $\xi$ candidate" under which $\xi$ events can fall. $\xi$ candidates must be defined entirely through detector response, with the goal of producing high sensitivity to underlying $\xi$ jets with reasonably good selectivity (i.e., mostly only $\xi$ jets register as $\xi$ candidates).

\section{C. $\xi$ candidates}

A detailed definition of the $\xi$ candidates category satisfying the demands stated above is best constructed by a team of experimental experts within the ATLAS and CMS collaborations deeply familiar with their detectors. However, it is likely that such a definition meeting the demands of sensitivity and selectivity will have several key characteristics which we would like to discuss here. We will then make illustrative estimates of the utility of a $\xi$ candidates definitions based on these characteristics.

We make use of MadGraph_aMC@NLO [25] simulations to produce our signal events at leading order with the lagrangian of Eq. (2), which are then hadronized via PYTHIA8 $[26,27]$. For our detector studies, we utilize the DELPHES [28] fast detector simulation framework with the default CMS card and FASTJET [29] for jet clustering algorithms.

To begin, one must have a cluster, established by standard techniques. One useful criterion to impose on the pre- $\xi$-candidate cluster is a strong isolation requirement against QCD activity within a small cone around the $\xi$-candidate system, reducing QCD backgrounds from decaying pions. Additional criteria for the definition must also appeal to the stoutness of the photon jet - there are two photons separated enough to not look like one photon, and that separation shows up as a larger-than-normal spatial spread among cells within the electromagnetic detector. Furthermore, vetoing on charged tracks eliminates electron-induced showers. Finally, recently established jet $n$-subjettiness algorithms [30] can be employed to select clusters that have discernible subjet structure compatible with two collimated photons. References [14,31] go into detail on the ability to use these and other, similar variables to separate $\xi$ candidates (called photon jets in these papers) from photons and QCD jets, but all of these considerations will be in play in the definitions below.

Our $\xi$-jet theory definition was for underlying twophoton clusters with $\Delta R$ separation in the range of
TABLE II. $\xi$ definition meant to capture underlying events with $0.025<\Delta R<0.25$. These objects are defined as a cone of radius $\Delta R=0.25$ about a central cluster in the EM calorimeter, centered on the highest energy pixel. The parameters are calculated over this full region.

\begin{tabular}{|c|c|c|c|}
\hline Variable & Definition & Cut & Reasoning \\
\hline $\log \theta_{J}$ & $\begin{array}{l}\text { Hadronic energy } \\
\text { fraction }\end{array}$ & $<-0.8$ & Exclude QCD and $\tau$ \\
\hline$N_{T}$ & $\begin{array}{c}\text { Number of } \\
\text { tracks }\end{array}$ & $=0$ & $\begin{array}{l}\text { Excludes single converted } \\
\text { photons and jet activity }\end{array}$ \\
\hline$\tau_{2} / \tau_{1}$ & $\begin{array}{l}\text { Ratio of 2- to 1- } \\
\text { subjettiness }\end{array}$ & $<0.3$ & $\begin{array}{l}\text { Selects events } \\
\text { with } 2 \text { subjets }\end{array}$ \\
\hline
\end{tabular}

0.04 to 0.4 . In addition, within the range of $0.025<\Delta R<$ 0.04 , there is a possibility of using electromagnetic shape variables to discern that the underlying event was likely not a single photon but certainly not clear enough to indicate the possibility of two photons. Nevertheless, our $\xi$-candidate list of criteria will be applicable for two-photon jets separations down to about $\Delta R \gtrsim 0.025$ and up to about $\Delta R \lesssim 0.25$. We will not discuss the range $0.25 \lesssim \Delta R \lesssim 0.4$ here because our understanding is that more traditional photon identification tools may be applicable to separate the photons just well enough to help discern signal from photon backgrounds.

Let us now turn to a more precise definition of $\xi$ candidates (underlying two-photon separation $0.025 \lesssim$ $\Delta R \lesssim 0.25)$. This regime targets events that have two photons in sufficiently close proximity that their cores overlap, thereby interfering with one another's identification procedures. This should appear as a cluster of energy in the electromagnetic (EM) calorimeter (ECAL), with no tracks or corresponding energy in the hadronic calorimeter, and high 2-subjettiness. We provide an example definition of $\xi$-candidate criteria in Table II. Below, in Fig. 3, we also show distributions of signal and background for QCD jets and $\xi$ jets. These distributions reproduce those of Refs. $[14,31]$ and show that $\xi$ jets can be separated from QCD backgrounds with high efficiency.

\section{Reconstructing $\xi$ jets and Higgs decays}

Now that we have precise definitions of photons and $\xi$ candidates, we can ask how well the Higgs boson signal can be reconstructed, especially in the case of its decay into one or more $\xi$ jets. Figure 4 shows the analysis flow of our reconstruction of $\xi$ jets using DELPHES fast detector simulation. Additional photons not covered by that flow, as well as electrons, muons, jets, etc., are identified and labeled by other analysis flows.

First, one must reconstruct the $\xi$ jets, which we attempt to do by following a strategy similar to Ref. [14]. The method is as follows. First, energy flow (eflow) objects [32] (composed of deposits in calorimeter cells) are clustered into jets using the anti-kt algorithm with $R=0.25$. 


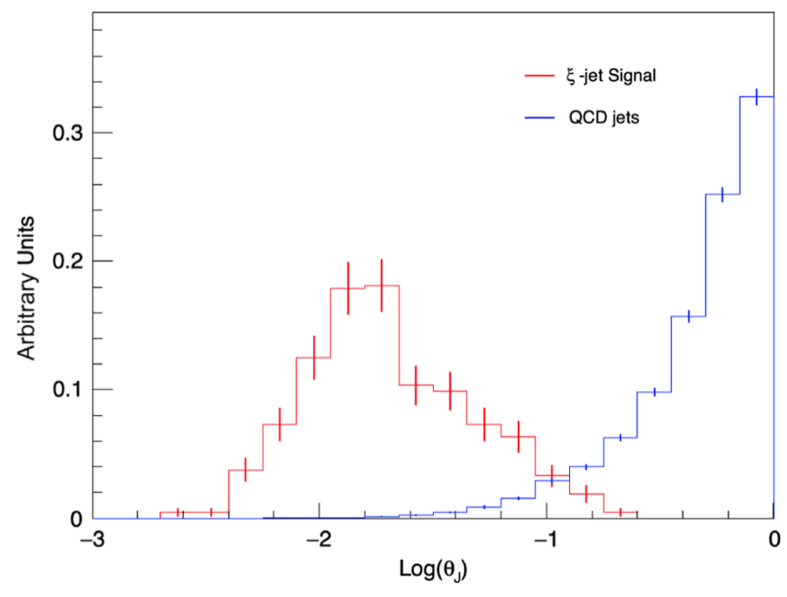

(a)

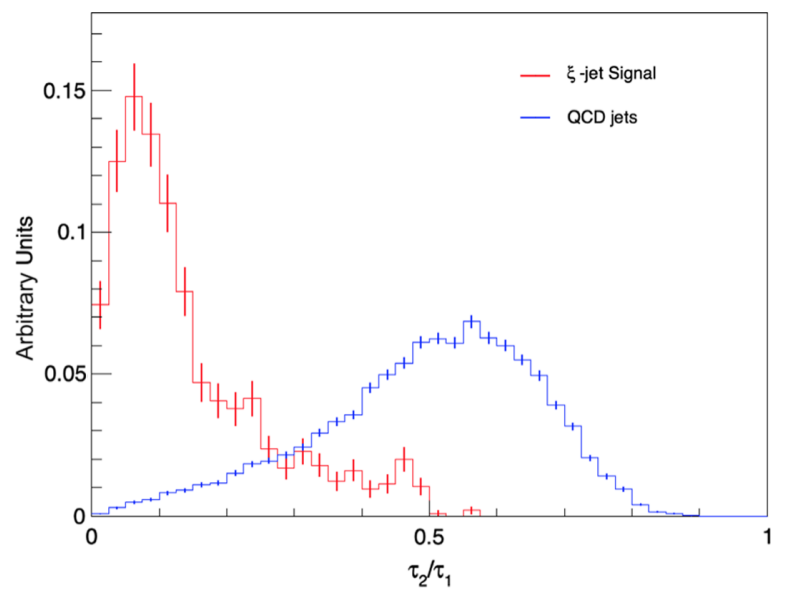

(b)

FIG. 3. Subset of kinematic variables useful for discriminating of $\xi$ jets (green) and QCD jets (blue) which are a major background. Here, $\theta_{J}$ is the hadronic energy fraction for a jet, and $\tau_{2} / \tau_{1}$ is the ratio of 2 -jettiness to 1 -jettiness, which is useful for picking out events with two subjets.

Then, we recluster those energy deposits that were found in each jet using the kt algorithm, which determines a recombination tree for the jets. This tree specifies the subjets at each level of recombination $N$ from $N=1$ (the full jet) to $N=$ the number of constituent eflow objects in the jet (no recombination). From here, we can compute the $N$-subjettiness variable for the jet for each $N$. This variable becomes small when the parameter $N$ is large enough to describe all of the relevant substructure of the jet. It is defined to be

$$
\tau_{N}=\frac{\Sigma_{k} p_{T_{k}} \times \min \left[\Delta R_{1, k}, \Delta R_{2, k}, \ldots, \Delta R_{N, k}\right]}{\Sigma_{k} p_{T_{k}} \times R},
$$

where $k$ runs over all the constituents of the jet, $p_{T_{k}}$ is the transverse momentum for the $k$ th constituent, and $R$ is the characteristic jet radius used in the original jet clustering algorithm.

After jet clustering is completed, we then check if a reconstructed $\xi$ candidate already contains a reconstructed

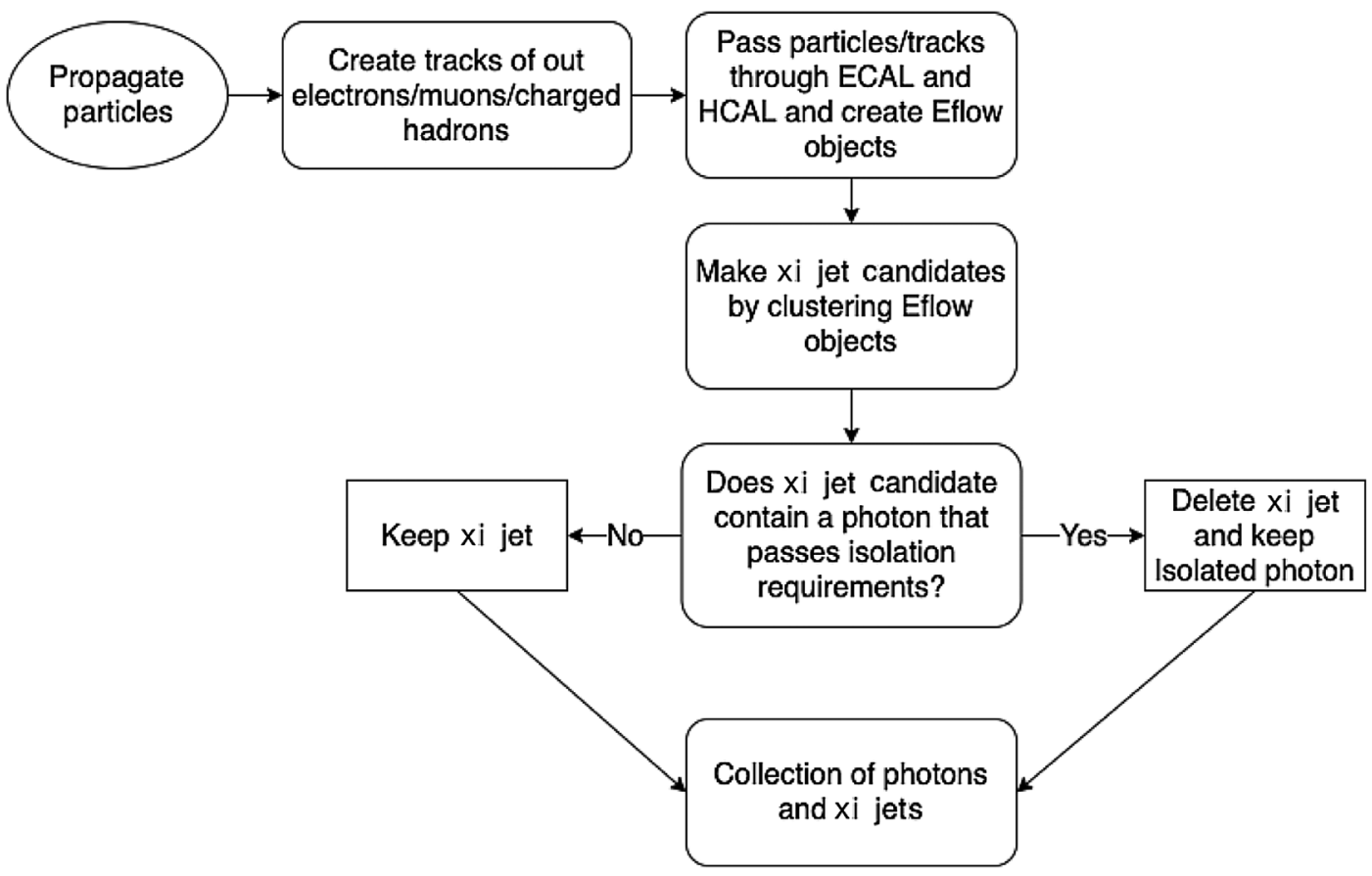

FIG. 4. The analysis flow of our reconstruction of $\xi$ jets using DELPHES fast detector simulation. Additional photons not covered by that flow, as well as electrons, muons, jets, etc., are identified and labeled by other analysis flows. 
photon. Reconstructed photons are composed of eflow objects originating from the ECAL which must pass isolation requirements (cuts on electromagnetic and hadronic activity within a cone around the photon). If a $\xi$ candidate contains an already reconstructed, isolated photon, then this $\xi$ candidate is deleted.

Before applying additional cuts, we would like to characterize the efficiency at which we reconstruct $\xi$ jets. To do this, we utilize DELPHES GenJet objects. GenJets are jets that are clustered, not with calorimeter cells or towers or eflow objects but with the actual generator-level particles. By utilizing GenJets, we can define "generated $\xi$ jets" and see at what rate we correctly reconstruct these.

GenJets are clustered with the same strategy as above, first with the anti-kt algorithm with $R=0.25$ and then reclustered with the kt algorithm. A GenJet is selected as a generated $\xi$ jet if it has 1) at most two photons with $p_{T}>0.5 \mathrm{GeV}$ and 2) no nonphotons with $p_{T}>0.5 \mathrm{GeV}$. Since our theoretical $\xi$ jets were defined as pairs of photons with $\Delta R$ between 0.04 and 0.4 , we throw out $\xi$ jets with $\Delta R<0.025$ as these will most likely be reconstructed as one photon.

Once a generated $\xi$ jet is identified, we loop over all reconstructed $\xi$ candidates and attempt to find a match. Matching is done by comparing the $\Delta R$ between the momentum of the generated and reconstructed jets. If $\Delta R_{\text {gen } / \text { reco }}<0.05$, we consider this jet as matched. We also require that the reconstructed $\xi$ jets pass a cut on the required hadronic energy fraction. This cut is that $\log \left(E_{\text {had }} / E_{\text {jet }}\right)<-0.8$. In Fig. 5, we show $\Delta R_{\text {gen/reco, }}$, which shows the level of matching between generated and reconstructed $\xi$ jets. It also serves as a check that this is independent of our model parameters.

Now, we would like to understand how often we can reconstruct the Higgs mass using our reconstructed photons and $\xi$ candidates. To simplify matters, we will choose $m_{\phi_{1}}=m_{\phi_{2}}$, which is equivalent to having only one light scalar in addition to the observed $h_{125}$. We scan over light

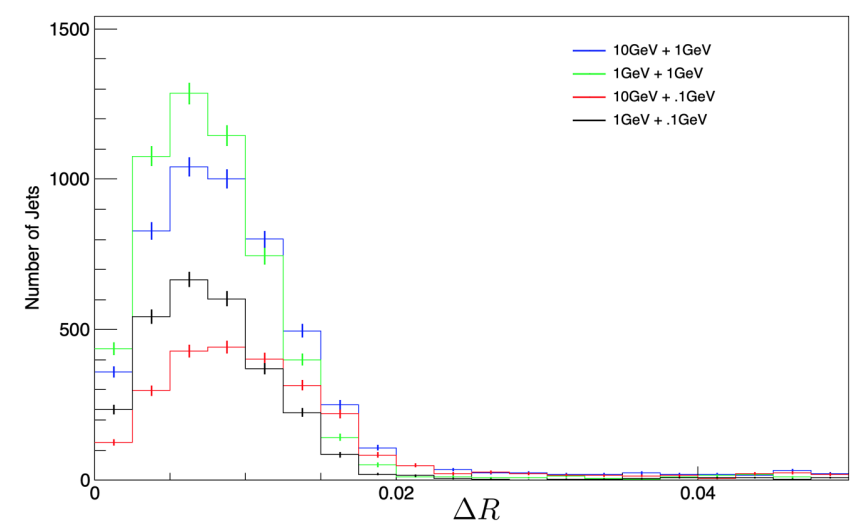

FIG. 5. $\Delta R$ between reconstructed and generated $\xi$ jets. Distribution is independent of our model parameters showing good matching between the two. scalar masses from $100 \mathrm{MeV}$ to $14 \mathrm{GeV}$. This range ensures we see a smooth transition between photon dominated decays and $\xi$-jet dominated decays. The following discussion can be generalized by choosing different masses for the light scalars. After reconstruction, we first collect all of our reconstructed objects, which for now are photons and $\xi$ candidates. We only require our reconstructed $\xi$ candidates to pass our hadronic energy fraction cut, otherwise no cuts (besides minimum $p_{T}$ cuts which are used for clustering). We then form all the possible subsets of this collection, which have between one and four objects (as at most the Higgs decayed into four separable photons). If one combination of $\xi$ candidates and photons yields an invariant mass within a $3 \mathrm{GeV}$ window around $125 \mathrm{GeV}(122 \mathrm{GeV}<$ $M_{\text {inv }}<128 \mathrm{GeV}$ ), then we consider this a match. Virtually no events contain multiple combinations of photons and $\xi$ jets which satisfy this requirement. We split each match into the following categories based on what number and type of objects make up the matching set:

(1) photons only: matches with two, three, or four photons

(2) photons $+\xi$ jets: matches with one photon + one $\xi$, two photons + one $\xi$

(3) $\xi$ jets only: events with two and only two $\xi$ jets

(4) $\xi$ jets inclusive: includes the photons $+\xi$-jets category as well as the $\xi$-jets-only category

(5) other combinations: any combination not included above

(6) all: any match in the accepted mass range.

Figure 6 then shows the efficiency of reconstructing the Higgs mass as a function of the light scalar mass. Several key observations can be made here. At very low scalar masses, photons only make up the dominant signal channel as the pairs of photons from $\phi$ decay are extremely collimated. From 100-300 MeV, the signal from photons + $\xi$ jets becomes the most efficient channel as one of the pairs of photons is collimated enough to form a $\xi$ jet. Immediately above $300 \mathrm{MeV}$, the signal from pairs of $\xi$ jets ( $\xi$ jets only) becomes an order of magnitude more efficient than the photon only channel and remains so until $6 \mathrm{GeV}$. Overall, searches including $\xi$ jets are more than an order of magnitude more efficient at reconstructing the Higgs from masses between $100 \mathrm{MeV}$ and $10 \mathrm{GeV}$.

Figure 6 shows that searches including $\xi$ jets would be invaluable if a light scalar connected to the gauge and Higgs sector as in Eq. (2) exists in nature. We would like to stress that, even though our analysis and definitions are quite simple, our results should be robust even after the introduction of more strict experimental search strategies and analysis cuts. It is interesting to compute how many such $\xi$-jet events one could expect for a given luminosity at the LHC. This is, of course, a function of the $\phi$ mass and the efficiency for reconstructing the $h_{125}$. To give an estimate, we can take the $m_{\phi}=2 \mathrm{GeV}$ point as an example. This has an efficiency for reconstruction of about 

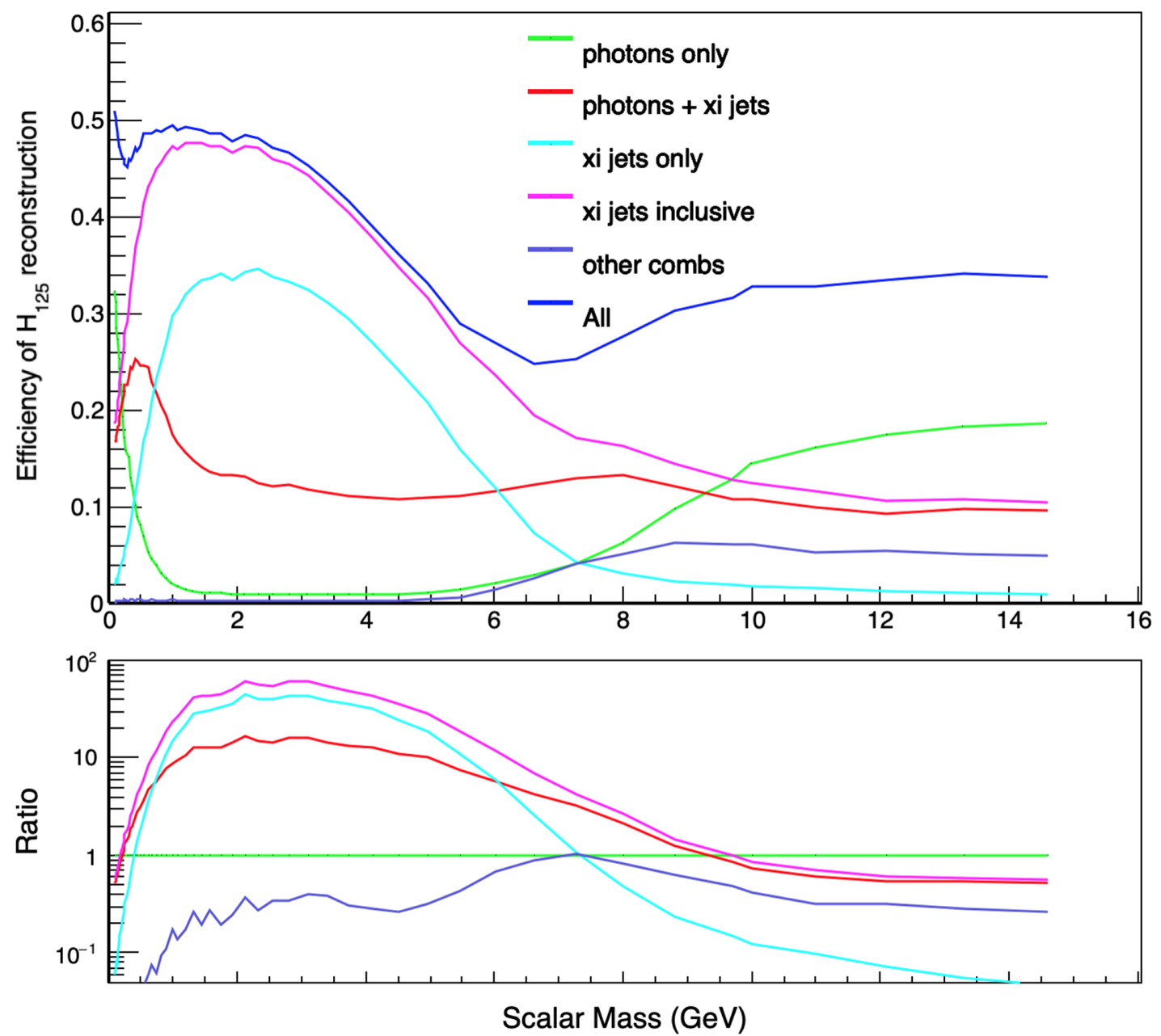

FIG. 6. Top: efficiency of $h_{125}$ reconstruction as a function of scalar mass split into different categories based on number of $\xi$ jets and photons. Bottom: ratio to only using photons. The reconstruction efficiency is more than an order of magnitude better when including $\xi$ jets (pink) over a wide range of masses from $100 \mathrm{MeV}$ to $10 \mathrm{GeV}$. More specifically, the two- $\xi$-jet channel dominates from the range of $300 \mathrm{MeV}$ to $6 \mathrm{GeV}$.

$50 \%$. If we take $\operatorname{Br}(h \rightarrow \phi \phi)=10^{-4}$ and an integrated luminosity of $300 \mathrm{fb}^{-1}$, this leaves us with about 7500 reconstructed events.

While a comprehensive study of standard model backgrounds is necessary for an experimental search, we can still make qualitative statements about discriminating $\xi$ jets from objects which fake $\xi$ jets. The largest backgrounds will be from $j j$ and $\gamma j$ production where a QCD jet fakes a $\xi$ jet. References [14,31] use a boosted decision tree based on energy and substructure variables to discriminate between QCD jets and photon jets. They quote a fake rate from QCD jets of $10^{-4}-10^{-5}$, though this fake rate is dependent on the rate at which one accidentally rejects $\xi$ jets. Additionally, the requirement that the invariant mass of the two $\xi$ candidates needs to fall within a $3 \mathrm{GeV}$ window of the $h_{125}$ mass lowers the background as well, as the rate for QCD jet production tends to fall at high invariant masses. Combined, these factors should allow for a bump hunt search for $\xi$ jets with high sensitivity.
Lastly, we would like to comment on our results in comparison to other results in the literature concerning similar rare Higgs decays to scalars. It is our understanding that these articles are principally concerned with only the separation of photon jets and nonphoton jets, e.g., analyzing purity and efficiency for photon jets vs single photons and other QCD related jets [14,31,33]. In contrast, we have tried to paint a picture which quantifies how such rare Higgs decays to $\phi_{i}$ can be missed if only traditional techniques involving photons are used. In providing a clear theoretical and experimental definition of a photon and a $\xi$ jet, we have been able to provide concrete branching fractions to states involving combinations of $\xi$ jets and photons only in terms of observables, which a detector actually measures. The extremely high ratio of events involving $\xi$ jets to events involving only photons serves as a testament to the need for such an analysis. We hope that this can guide future experimental inquiries into models such as those described in Sec. II. 


\section{CONCLUSION}

The discovery of the Higgs boson has lent strong support to the Standard Model but also has allowed us to search for new avenues along which to extend it. In this work, we have investigated exotic decays of the $125 \mathrm{GeV}$ Higgs boson into light scalars which as of yet may be missed via current analysis techniques. We have discussed, first theoretically and then experimentally, a new object dubbed a $\xi$ jet, which could play a pivotal role in the discovery of any light scalars minimally coupled to the standard model Higgs and to photons as in Eq. (2). If experimentalists are able to identify and reconstruct $\xi$ jets, these new objects could be strong evidence for an extended Higgs sector and beyond the Standard Model physics.

\section{ACKNOWLEDGMENTS}

We thank Advanced Research Computing at the University of Michigan, Ann Arbor for their computational resources as well as D. Amidei, C. Hayes, R. Hyneman, and T. Schwarz for helpful conversations on these issues. This work was supported in part by the DOE under Grant No. DE-SC0007859. B. Sheff is supported by the NSF GRFP program, and N. Steinberg is supported by a fellowship from the Leinweber Center for Theoretical Physics.
[1] A. M. Sirunyan et al. (CMS Collaboration), Search for invisible decays of a Higgs boson produced through vector boson fusion in proton-proton collisions at $\sqrt{s}=13 \mathrm{TeV}$, Phys. Lett. B 793, 520 (2019).

[2] M. Aaboud et al. (ATLAS Collaboration), Measurements of Higgs boson properties in the diphoton decay channel with $36 \mathrm{fb}^{-1}$ of $p p$ collision data at $\sqrt{s}=13 \mathrm{TeV}$ with the ATLAS detector, Phys. Rev. D 98, 052005 (2018).

[3] G. Belanger, B. Dumont, U. Ellwanger, J. Gunion, and S. Kraml, Global fit to Higgs signal strengths and couplings and implications for extended Higgs sectors, Phys. Rev. D 88, 075008 (2013).

[4] G. Aad et al. (ATLAS Collaboration), Combined measurements of Higgs boson production and decay using up to $80 \mathrm{fb}^{-1}$ of proton-proton collision data at $\sqrt{s}=13 \mathrm{TeV}$ collected with the ATLAS experiment, Phys. Rev. D 101, 012002 (2020).

[5] G. Aad et al. (ATLAS, CMS Collaborations), Measurements of the Higgs boson production and decay rates and constraints on its couplings from a combined ATLAS and CMS analysis of the LHC pp collision data at $\sqrt{s}=7$ and $8 \mathrm{TeV}$, J. High Energy Phys. 08 (2016) 045.

[6] D. Curtin et al., Exotic decays of the $125 \mathrm{GeV}$ Higgs boson, Phys. Rev. D 90, 075004 (2014).

[7] G. Aad et al. (ATLAS Collaboration), A search for the dimuon decay of the Standard Model Higgs boson with the ATLAS detector, Phys. Lett. B 812, 135980 (2021).

[8] G. Aad et al. (ATLAS Collaboration), Combination of searches for Higgs boson pairs in $p p$ collisions at $\sqrt{s}=13 \mathrm{TeV}$ with the ATLAS detector, Phys. Lett. B 800, 135103 (2020).

[9] B. A. Dobrescu, G. L. Landsberg, and K. T. Matchev, Higgs boson decays to $C P$ odd scalars at the Tevatron and beyond, Phys. Rev. D 63, 075003 (2001).

[10] P. Draper and D. McKeen, Diphotons from Tetraphotons in the Decay of a $125 \mathrm{GeV}$ Higgs at the LHC, Phys. Rev. D 85, 115023 (2012).

[11] A. Collaboration, Search for new phenomena in events with at least three photons collected in $p p$ collisions at $\sqrt{s}=8 \mathrm{TeV}$ with the ATLAS detector, Eur. Phys. J. C 76, 210 (2016).

[12] S. Chang, P. J. Fox, and N. Weiner, Visible Cascade Higgs Decays to Four Photons at Hadron Colliders, Phys. Rev. Lett. 98, 111802 (2007).

[13] M. Tanabashi et al. (Particle Data Group), Review of particle physics, Phys. Rev. D 98, 030001 (2018).

[14] S. D. Ellis, T. S. Roy, and J. Scholtz, Phenomenology of photon-jets, Phys. Rev. D 87, 014015 (2013).

[15] ATLAS Collaboration, Search for a Higgs boson decaying to four photons through light $C P$-odd scalar coupling using $7 \mathrm{TeV} p p$ collision data taken with ATLAS detector at the LHC, CERN Report No. ATLAS-CONF-2012-079, 2012, http://cds.cern.ch/record/1460391.

[16] H. A. Chawdhry, M. Czakon, A. Mitov, and R. Poncelet, NNLO QCD corrections to three-photon production at the LHC, J. High Energy Phys. 02 (2020) 057.

[17] M. Aaboud et al. (ATLAS Collaboration), A search for pairs of highly collimated photon-jets in $p p$ collisions at $\sqrt{s}=13 \mathrm{TeV}$ with the ATLAS detector, Phys. Rev. D 99, 012008 (2019).

[18] A. Collaboration, Electron and photon performance measurements with the ATLAS detector using the 2015-2017 LHC proton-proton collision data, J. Instrum. 14, P12006 (2019).

[19] T. Robens and T. Stefaniak, Status of the Higgs singlet extension of the standard model after LHC run 1, Eur. Phys. J. C 75, 104 (2015).

[20] V. Barger, P. Langacker, M. McCaskey, M. J. RamseyMusolf, and G. Shaughnessy, LHC phenomenology of an extended Standard Model with a real scalar singlet, Phys. Rev. D 77, 035005 (2008).

[21] T. Robens, T. Stefaniak, and J. Wittbrodt, Two-realscalar-singlet extension of the SM: LHC phenomenology and benchmark scenarios, Eur. Phys. J. C 80, 151 (2020).

[22] D. López-Val and T. Robens, $\Delta \mathrm{r}$ and the W-boson mass in the singlet extension of the standard model, Phys. Rev. D 90, 114018 (2014). 
[23] C. Csaki, R. T. D’Agnolo, M. Geller, and A. Ismail, Crunching Dilaton, Hidden Naturalness, Phys. Rev. Lett. 126, 091801 (2021).

[24] T. A. Aaltonen et al. (CDF Collaboration), First Search for Exotic Z Boson Decays into Photons and Neutral Pions in Hadron Collisions, Phys. Rev. Lett. 112, 111803 (2014).

[25] J. Alwall, R. Frederix, S. Frixione, V. Hirschi, F. Maltoni, O. Mattelaer, H. S. Shao, T. Stelzer, P. Torrielli, and M. Zaro, The automated computation of tree-level and next-to-leading order differential cross sections, and their matching to parton shower simulations, J. High Energy Phys. 07 (2014) 079.

[26] T. Sjostrand, S. Mrenna, and P.Z. Skands, PYTHIA 6.4 Physics and Manual, J. High Energy Phys. 05 (2006) 026.

[27] T. Sjostrand, S. Mrenna, and P.Z. Skands, A brief introduction to PYTHIA 8.1 Comput. Phys. Commun. 178, 852 (2008).

[28] J. de Favereau, C. Delaere, P. Demin, A. Giammanco, V. Lemaître, A. Mertens, and M. Selvaggi (DELPHES 3
Collaboration), DELPHES 3, A modular framework for fast simulation of a generic collider experiment, J. High Energy Phys. 02 (2014) 057.

[29] M. Cacciari, G. Salam, and G. Soyez, FastJet user manual, Eur. Phys. J. C 72, 1896 (2012).

[30] J. Thaler and K. Van Tilburg, Identifying Boosted Objects with N-subjettiness, J. High Energy Phys. 03 (2011) 015.

[31] A. Chakraborty, A. M. Iyer, and T. S. Roy, A Framework for Finding Anomalous Objects at the LHC, Nucl. Phys. B932, 439 (2018).

[32] CMS Collaboration, Particle-Flow Event Reconstruction in CMS and Performance for Jets, Taus, and MET, CERN Report No. CMS-PAS-PFT-09-001, 2009, https://cds.cern .ch/record/1194487.

[33] E. Hall and J. Thaler, Photon isolation and jet substructure, J. High Energy Phys. 09 (2018) 164. 